\title{
低温烧结陶瓷 $\mathrm{BiMg}_{2} \mathrm{VO}_{6}$ 的微波介电性能
}

\author{
谢会东，席海红，李飞，陈 超 \\ (西安建筑科技大学 理学院, 西安 710055)
}

摘 要: 通过传统的固相反应方法制备了低温烧结微波介质陶瓷 $\mathrm{BiMg}_{2} \mathrm{VO}_{6}$, 研究了该陶瓷与银的化学兼容性、物 相、形貌及在 $720 \sim 840^{\circ} \mathrm{C}$ 内的密度和微波介电性质，并测试了陶瓷的红外反射光谱。结果表明：陶瓷在 $780^{\circ} \mathrm{C}$ 条件 下与银共烧不发生反应，相对密度大于 $93.8 \%$ 。在 $780^{\circ} \mathrm{C}$ 条件下烧结 $2 \mathrm{~h}$ 得到的陶瓷具有最好的微波介电性能: 介 电常数为 $13.4, Q \times f$ 值为 $15610 \mathrm{GHz}(f=8.775 \mathrm{GHz})$, 温度系数为 $-87.2 \times 10^{-6} /{ }^{\circ} \mathrm{C}$ 。红外反射谱数据处理显示, $\mathrm{BiMg}_{2} \mathrm{VO}_{6}$ 的光频介电常数 $\varepsilon_{\infty}=3.4$, 微波频段的外推值为 $13.5 \circ \mathrm{BiMg}_{2} \mathrm{VO}_{6}$ 陶瓷好的微波介电性能和低的烧结温度, 使其有望用作新的低温共烧陶瓷。

关 键 词: $\mathrm{BiMg}_{2} \mathrm{VO}_{6}$; 微波介电; 烧结性; 陶瓷

中图分类号: TQ174 文献标识码: A

\section{Microwave Dielectric Properties of $\mathrm{BiMg}_{2} \mathrm{VO}_{6}$ Ceramic with Low Sintering Temperature}

\author{
XIE Hui-Dong, XI Hai-Hong, LI Fei, CHEN Chao \\ (School of Science, Xi'an University of Architecture and Technology, Xi'an 710055, China)
}

\begin{abstract}
A low firing microwave dielectric ceramic $\mathrm{BiMg}_{2} \mathrm{VO}_{6}$ was prepared via conventional solid state reaction method. The chemical compatibility, phase, morphology, density and microwave dielectric properties of the ceramic in the sintering temperature range of $720 \sim 840^{\circ} \mathrm{C}$ were studied. The infrared reflectivity spectra of the ceramic were measured. Results showed that the $\mathrm{BiMg}_{2} \mathrm{VO}_{6}$ ceramics did not react with $\mathrm{Ag}$ at $780^{\circ} \mathrm{C}$ and the relative densities of the samples were greater than $93.8 \%$ at every sintering temperature conditions. Ceramic sintered at $780^{\circ} \mathrm{C}$ for $2 \mathrm{~h}$ showed the optimum microwave dielectric properties with permittivity of $13.34, Q \times f$ value of $15610 \mathrm{GHz}(f=8.775 \mathrm{GHz})$ and temperature coefficient value of $-87.2 \times 10^{-6} /{ }^{\circ} \mathrm{C}$. The optical frequency permittivity was 3.4 and the extrapolated value to microwave frequency was 13.5. The optimum microwave dielectric properties and low sintering temperature of $\mathrm{BiMg}_{2} \mathrm{VO}_{6}$ ceramic enable it a promising candidate for low temperature co-fired ceramic applications.
\end{abstract}

Key words: $\mathrm{BiMg}_{2} \mathrm{VO}_{6}$; microwave dielectric properties; sintering behavior

微波介质陶瓷广泛用于制造介质谐振器、滤波 器、介质基片、天线等微波器件中。随着无线通信 和卫星工业等微波设备向小型化、集成化以及民用 方向发展，利用低温共烧陶瓷(LTCC)技术制造片式 多层微波器件已成为当今的研究热点 ${ }^{[1-4]}$ 。对 LTCC
材料的要求是: 较低烧结温度(低于 $\mathrm{Ag} 、 \mathrm{Al} 、 \mathrm{Cu}$ 、 $\mathrm{Au}$ 等常用金属的熔点), 适当的介电常数 $\left(\varepsilon_{\mathrm{r}}\right)$ 、高的 品质因子 $(Q \times f)(f$ 为谐振频率, $Q$ 为 $1 /$ 介电损耗 $)$, 近 零的谐振频率温度系数 $\left(\mathrm{TCF} \approx 0 \mathrm{ppm} /{ }^{\circ} \mathrm{C}\right)$ 、与金属电 极的烧结匹配、低成本(不含或者含有少量贵金属)、 
环保(至少无铅，尽量不含有毒原材料) ${ }^{[5-6]}$ 。

LTCC 技术的关键是研制能与 $\mathrm{Ag} 、 \mathrm{Cu}$ 等电极共 烧的微波介质陶瓷。由于氧化铋的熔点低 $\left(825^{\circ} \mathrm{C}\right)$, 铋 基陶瓷一般具有低或超低的烧结温度, 是研究的热 点 $^{[7-10]}$ 。其次, 需要考虑提高品质因子。通常 $Q \times f$ 由本征损耗和非本征损耗构成。本征损耗由晶格振 动声学模造成; 非本征损耗受许多缺陷如晶界、粒 径、二次生长和气孔的影响 ${ }^{[11]}$, 且可以通过优化烧 结工艺来改善。利用 Kramers-Kronig(KK)变换关系 式，通过拟合红外反射谱的方法并外推至微波频段， 得到的介电常数实部应该与微波频段下的实测值吻 合, 得到的介电损耗(即虚部与实部的比值)一般认 为是本征介电损耗 ${ }^{[12]}$ 。

钒酸二镁铋 $\left(\mathrm{BiMg}_{2} \mathrm{VO}_{6}\right)$ 晶体属正交晶系，是 $\mathrm{BiA}_{2} \mathrm{MO}_{6}$ 系列中的一员 $(\mathrm{A}=\mathrm{Mg}, \mathrm{Ca}, \mathrm{Cd}, \mathrm{Pb}, \mathrm{Cu}, \mathrm{Zn}$; $\mathrm{M}=\mathrm{P}, \mathrm{As}, \mathrm{V})$, 最早由 Sleight 课题组制备并报道了结

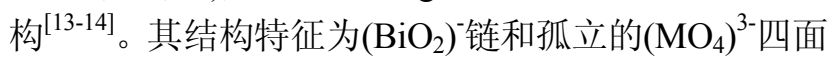
体, $\mathrm{A}$ 为 5 配位, 通常用固相法在空气气氛中制备。 $\mathrm{BiMg}_{2} \mathrm{VO}_{6}$ 具有铁电性, 可作黄色颜料 ${ }^{[15]}$ 。目前文献 对其应用报道较少, Guo 等 ${ }^{[16]}$ 用其作为光催化的载 体材料。到目前为止, 还没有关于 $\mathrm{BiMg}_{2} \mathrm{VO}_{6}$ 陶瓷制 备和微波介电性能的报道。本研究用常规固相法合 成了低介电常数的 $\mathrm{BiMg}_{2} \mathrm{VO}_{6}$ 陶瓷, 研究了不同烧 结温度下陶瓷的微波介电性能以及与金属银粉的共 烧性，并对陶瓷的红外反射光谱进行了测量和拟合。

\section{1 实验方法}

\section{1 样品制备}

采用分析纯的 $\mathrm{Bi}_{2} \mathrm{O}_{3}(99.0 \%) 、 \mathrm{MgO}(98.5 \%)$ 和 $\mathrm{V}_{2} \mathrm{O}_{5}(99.0 \%)$ 为原料, 按 $\mathrm{BiMg}_{2} \mathrm{VO}_{6}$ 的化学计量比配 料, 总重约 $25 \mathrm{~g}$, 以无水乙醇为介质研磨 $2 \mathrm{~h}$, 挥发 乙醇, $80^{\circ} \mathrm{C}$ 烘干; 于 $650^{\circ} \mathrm{C}$ 预烧 $6 \mathrm{~h}$ 后, 二次干法研 磨 $0.5 \mathrm{~h}, 80^{\circ} \mathrm{C}$ 烘干; 粉碎过篮后加入 $5 \mathrm{wt} \%$ 的 PVA 溶 液造粒, 再压制成 $\phi 12 \mathrm{~mm} \times 5 \mathrm{~mm}$ 的圆柱体, 压力为 $150 \mathrm{MPa} ; 550^{\circ} \mathrm{C}$ 排胶后, 在 $720 \sim 840^{\circ} \mathrm{C}$ 烧结 $2 \mathrm{~h}$, 用程 序控温炉以 $3{ }^{\circ} \mathrm{C} / \mathrm{min}$ 升温烧制成淡黄色陶瓷样品。为 了进一步研究 $\mathrm{BiMg}_{2} \mathrm{VO}_{6}$ 与金属 $\mathrm{Ag}$ 的化学兼容性, 用 $\mathrm{Ag}$ 粉与合成的 $\mathrm{BiMg}_{2} \mathrm{VO}_{6}$ 粉混合后压成陶瓷素坏在 $780^{\circ} \mathrm{C}$ 共烧 $4 \mathrm{~h}$, 所用 $\mathrm{Ag}$ 粉质量占总重的 $20 \%$ 。

\section{2 样品测试}

采用 Rigaku 公司生产的 D/MAX-2400 型 X 射 线衍射仪对样品进行 XRD 分析; 采用日本 JEOL 公司生产的 JSM-6460 扫描电子显微镜(SEM)对陶 瓷表面做形貌检测; 采用阿基米德排水法测量陶瓷
的密度; 采用国家同步辐射实验室的 Bruker IFS 66v FTIR 谱仪测量室温下的红外反射谱, 测试范围 为 $50 \sim 7000 \mathrm{~cm}^{-1}$ 。采用 Agilent 公司生产的 $8720 \mathrm{ES}$ 型网络分析仪及 9023 型温度室(闭式腔法)测量样品 的微波介电性能及谐振频率温度系数。其中, 样品 温度系数的测量范围为 $25 \sim 85^{\circ} \mathrm{C}$ 。谐振频率温度系 数按下式计算:

$$
\mathrm{TCF}=\frac{f_{85}-f_{25}}{f_{25}(85-25)} 10^{-6} /{ }^{\circ} \mathrm{C}
$$

式中 $f_{85} 、 f_{25}$ 分别为 $85^{\circ} \mathrm{C} 、 25^{\circ} \mathrm{C}$ 时的谐振频率。

\section{2 结果与讨论}

\section{$2.1 X R D$ 物相分析}

图 1 为 $780^{\circ} \mathrm{C}$ 烧结 $4 \mathrm{~h}$ 的 $\mathrm{BiMg}_{2} \mathrm{VO}_{6}$ 陶瓷和共烧 陶瓷的 XRD 图谱。由图 1(a)可知，所合成的陶瓷图 谱与 $\mathrm{BiMg}_{2} \mathrm{VO}_{6}$ 的标准衍射卡片(JCPDS 号: 48-0195) 相比完全一致, 无杂相, 产物为正交相, 空间群 $\mathrm{Cmcm}$, 晶胞参数 $a=0.7914 \mathrm{~nm}, b=1.2246 \mathrm{~nm}, c=$ $0.5444 \mathrm{~nm}$ 。由图 1(b)可知, 对共烧陶瓷样品, 图谱中 只有 $\mathrm{BiMg}_{2} \mathrm{VO}_{6}$ 相和金属银的峰(JCPDS 号: 04- 0783), 没有额外的杂相峰存在, 表明 $\mathrm{BiMg}_{2} \mathrm{VO}_{6}$ 陶瓷在 $780^{\circ} \mathrm{C}$ 与 $\mathrm{Ag}$ 共烧, 不发生反应。

\section{2 形貌分析}

图 2 为在不同温度下烧结 $2 \mathrm{~h}$ 的 $\mathrm{BiMg}_{2} \mathrm{VO}_{6}$ 陶瓷 的二次电子电镜照片和共烧陶瓷的背散射电镜照 片。从图 2(a)看出, $720^{\circ} \mathrm{C}$ 烧结的陶瓷有气孔存在, 从 图 2(b e) 看到, 在 740 840 ${ }^{\circ} \mathrm{C}$ 下烧结得到的陶瓷均致 密均匀, 晶界明显, 几乎没有气孔。说明 $\mathrm{BiMg}_{2} \mathrm{VO}_{6}$ 瓷晶粒尺寸越大。 $840^{\circ} \mathrm{C}$ 大颗粒晶粒形状从近等轴状

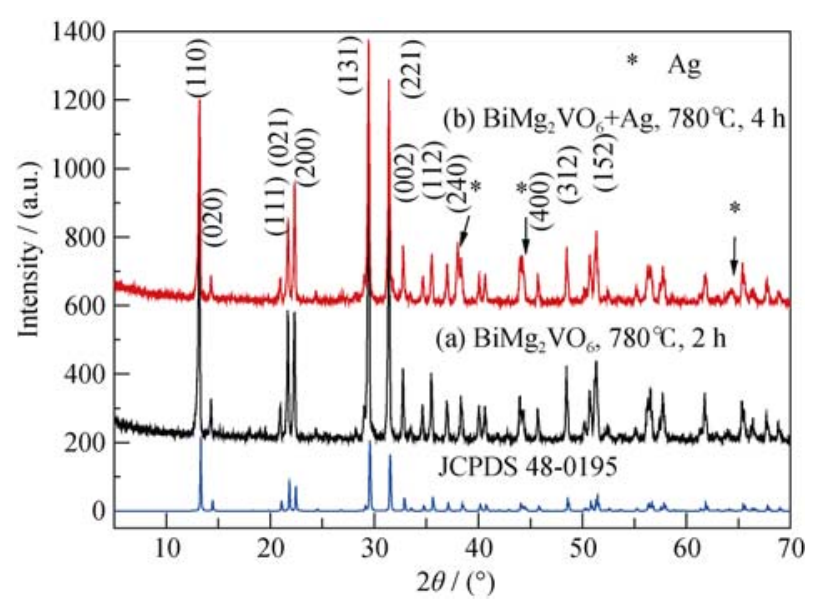

图 $1 \mathrm{BiMg}_{2} \mathrm{VO}_{6}$ 陶瓷以及共烧陶瓷的 XRD 图谱

Fig. 1 X-ray diffraction patterns of $\mathrm{BiMg}_{2} \mathrm{VO}_{6}$ ceramic and co-fired ceramic 
组分易于烧结成瓷。此外烧结温度越高, 所得到的陶 变成长条状, 这表明温度的升高有助于晶粒定向生 长。从图 2(f) 可以看出, 共烧陶瓷主要有浅颜
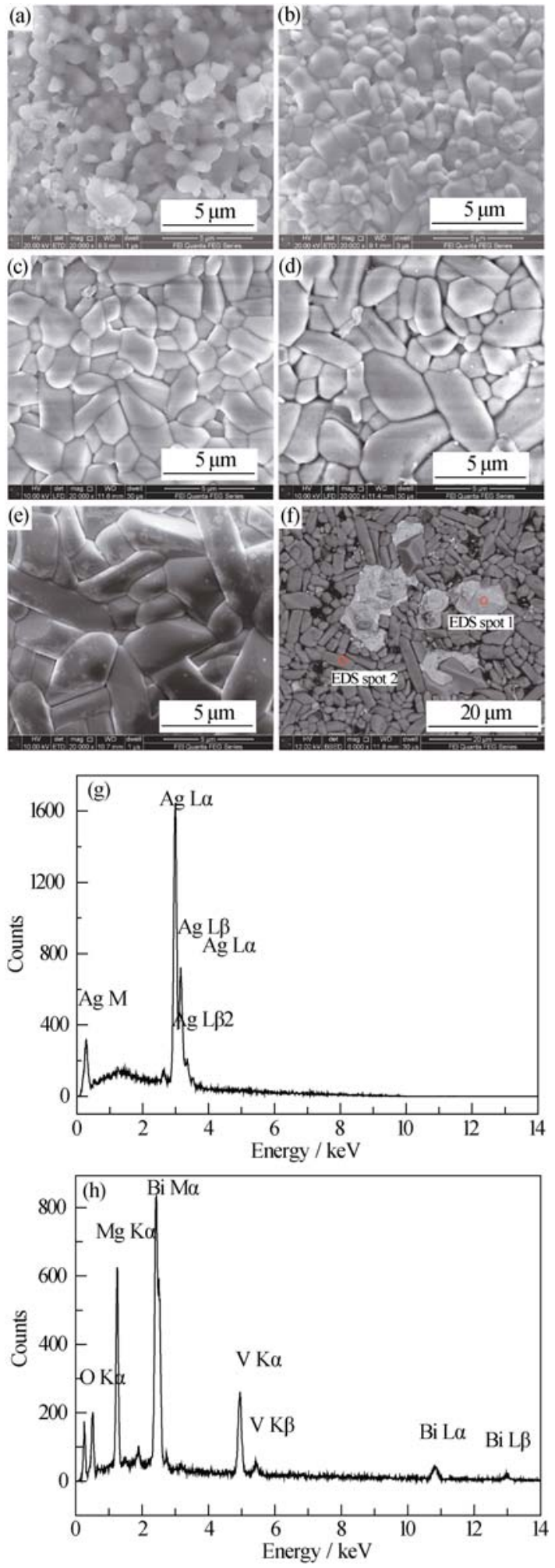

图 2 不同温度烧结 $2 \mathrm{~h}$ 所得 $\mathrm{BiMg}_{2} \mathrm{VO}_{6}$ 陶瓷表面的二次电子 照片(a e) 和背散射电子照片(f)及其 EDS 分析结果( $\mathrm{g}, \mathrm{h}$ )

Fig. 2 Secondary electron images (a-e), backscattered electron image (f) and its energy dispersive spectra $(g, h)$ of the as-co-fired $\mathrm{BiMg}_{2} \mathrm{VO}_{6}$ surfaces sintered at $720^{\circ} \mathrm{C}(\mathrm{a}), 740^{\circ} \mathrm{C}(\mathrm{b})$, $760^{\circ} \mathrm{C}(\mathrm{c}), 780^{\circ} \mathrm{C}(\mathrm{d})$ and $840^{\circ} \mathrm{C}(\mathrm{e})$ for $2 \mathrm{~h}$
色规则外形的、数微米大小的晶粒和不规则白色物 质组成，能量色散分析(EDS)结果证实位于 spot 1 的白色物质属于纯银, 见图 $2(\mathrm{~g})$, 位于 spot 2 的灰 色晶粒属于 $\mathrm{BiMg}_{2} \mathrm{VO}_{6}$, 见图 2(h)。共烧陶瓷背散 射结果结合 XRD 分析结果, 推测 $\mathrm{BiMg}_{2} \mathrm{VO}_{6}$ 在共 烧阶段没有与银粉反应生成化合物。由于 $\mathrm{BiMg}_{2} \mathrm{VO}_{6}$ 的烧结温度低于银的熔点 $\left(961^{\circ} \mathrm{C}\right)$, 且与 银有相容性。因此 $\mathrm{BiMg}_{2} \mathrm{VO}_{6}$ 有望用作新的低温共 烧陶瓷材料。

\section{3 微波介电性能}

图 3 为不同温度下烧结的 $\mathrm{BiMg}_{2} \mathrm{VO}_{6}$ 陶瓷的实 测密度和相对密度。由图可以看出, $\mathrm{BiMg}_{2} \mathrm{VO}_{6}$ 陶瓷 的密度随着烧结温度升高呈现先增大后减小的趋势, 在 $780^{\circ} \mathrm{C}$ 达到最大实测密度为 $5.00 \mathrm{~g} / \mathrm{cm}^{3}$ 。这是因为 随着温度的升高，陶瓷的孔隙率会逐渐减小，但在 达到最大密度后, 随着烧结温度的进一步增加, 由 于二次晶粒生长, 密度通常会略有降低 ${ }^{[11]}$ 。从相对 密度来看, 所有温度下 $\mathrm{BiMg}_{2} \mathrm{VO}_{6}$ 陶瓷相对密度均 不低于 $93.8 \%$ ，最大的相对密度为 $98.1 \%$ ，这说明 $\mathrm{BiMg}_{2} \mathrm{VO}_{6}$ 成分容易烧结成瓷。

图 4 为不同温度下烧结的 $\mathrm{BiMg}_{2} \mathrm{VO}_{6}$ 陶瓷的微 波介电性能。介电常数一般和相对密度的变化规律 相似：随着烧结温度的升高，微波介电常数先增大 后减小, 在 $780^{\circ} \mathrm{C}$ 达到极大值。这是因为介电常数 受单位体积中的极化粒子数影响很大，陶瓷的密 度越高, 单位体积中的可极化粒子数越多, 所以介 电常数增大 ${ }^{[17]}$ 。对 $\mathrm{BiMg}_{2} \mathrm{VO}_{6}, Q \times f$ 也显示和介电常 数相似的变化规律, 即先增大后减小, 并在 $780^{\circ} \mathrm{C}$ 达 到极大值: 介电常数 $13.4, Q \times f$ 值为 $15610 \mathrm{GHz}$ $(f=8.775 \mathrm{GHz})$ 。在该烧结条件下从方程(1)计算得到 的温度系数为 $-87.2 \times 10^{-6} /{ }^{\circ} \mathrm{C}$ 。一般来说, 大的晶粒

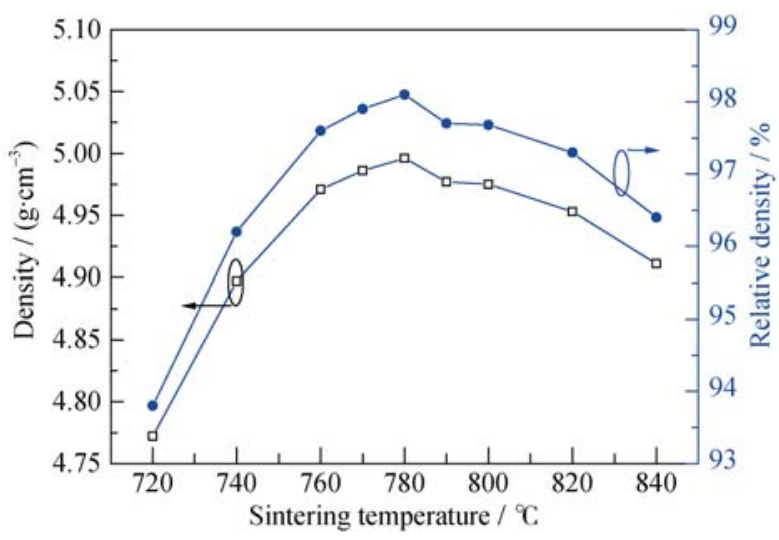

图 3 不同温度下烧结的 $\mathrm{BiMg}_{2} \mathrm{VO}_{6}$ 陶瓷的实测密度和相对 密度

Fig. 3 Apparent and relative densities of $\mathrm{BiMg}_{2} \mathrm{VO}_{6}$ ceramics as a function of sintering temperature 


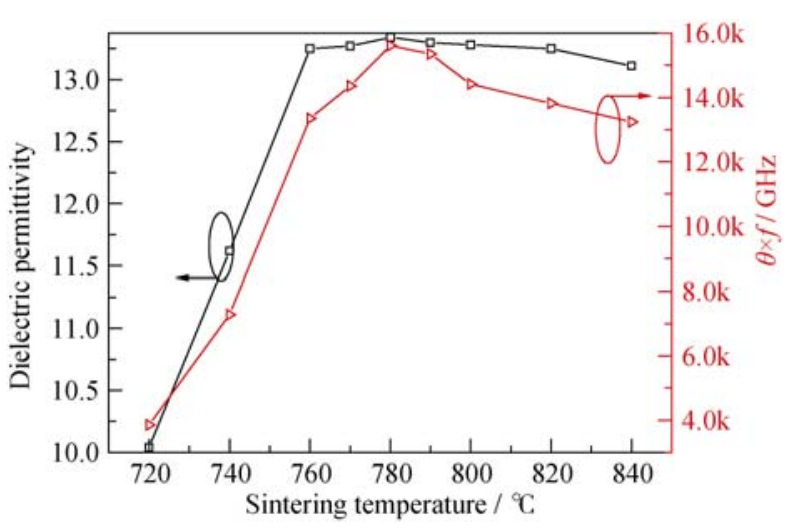

图 4 不同温度下烧结的 $\mathrm{BiMg}_{2} \mathrm{VO}_{6}$ 陶瓷的微波介电性能 Fig. 4 Microwave dielectric properties (permittivity, $Q \times f$ value) of $\mathrm{BiMg}_{2} \mathrm{VO}_{6}$ ceramics as a function of sintering temperature

尺寸、高的密度和均一的晶粒结构具有高的 $Q \times f$ 值。然而, 第二相和异常晶粒生长会恶化体系的 $Q$ $\times f$ 值。这在图 2 的扫描电镜照片中得到证实。

$\mathrm{BiMg}_{2} \mathrm{VO}_{6}$ 陶瓷在 $840^{\circ} \mathrm{C}$ 有异常长大的晶粒, 造成该 温度下介电损耗的增加。因此, 高 $Q \times f$ 的微波介质 陶瓷只能在一个狭窄的烧结温度下实现 ${ }^{[11]}$ 。

\section{4 远红外反射谱}

为了了解 $\mathrm{BiMg}_{2} \mathrm{VO}_{6}$ 陶瓷的微波介电性能, 对 其红外反射谱使用经典谐振子模型, 使用 Reffit 软 件进行拟合 ${ }^{[18]}$ 。在这一模型中, 复介电常数公式为:

$$
\varepsilon^{*}(\omega)=\varepsilon_{\infty}+\sum_{j=1}^{n} \frac{\omega_{\mathrm{pj}}^{2}}{\omega_{\mathrm{oj}}^{2}-\omega^{2}-i \gamma_{\mathrm{j}} \omega}
$$

式中, $\varepsilon *(\omega)$ 为复介电常数; $\varepsilon_{\infty}$ 为光频下电子 极化对介电常数的贡献; $n$ 为光学横模模式的数量; $\gamma_{\mathrm{j}} 、 \omega_{\mathrm{oj}}$ 和 $\omega_{\mathrm{pj}}$ 分别为第 $j$ 个洛仑兹谐振模式的阻尼 系数、光学横模频率以及振动强度。反射率 $R(\omega)$ 与复介电常数的关系为:

$$
R(\omega)=\left|\frac{1-\sqrt{\varepsilon^{*}(\omega)}}{1+\sqrt{\varepsilon^{*}(\omega)}}\right|^{2}
$$

对红外反射谱的拟合结果和实测结果如图 5(c) 所示，根据拟合数据得到的介电常数的实部和虚部 如图 5(a,b)所示。对 $\mathrm{BiMg}_{2} \mathrm{VO}_{6}$, 可观测的振动模式 数目为 19 , 拟合得到的 $\mathrm{BiMg}_{2} \mathrm{VO}_{6}$ 材料的光频介电 常数为 3.4 , 对微波频段的介电常数贡献较小, 距离 微波频段较近的远红外频段对其介电极化贡献则较 大。随着频率的升高, 声学模式振动提供的极化贡 献逐渐减小。将图 5(b) 外推至微波频段得到 $\mathrm{BiMg}_{2} \mathrm{VO}_{6}$ 材料的介电常数为 13.5 , 而实际测量值 为 $13.4(f=8.775 \mathrm{GHz})$, 略高于计算值。将图 5(a)外 推至微波频段, 得到微波频段 $8.775 \mathrm{GHz}$ 的复介电 常数的虚部为 $2.8 \times 10^{-3}$, 由此计算得出的 $Q \times f$ 值为

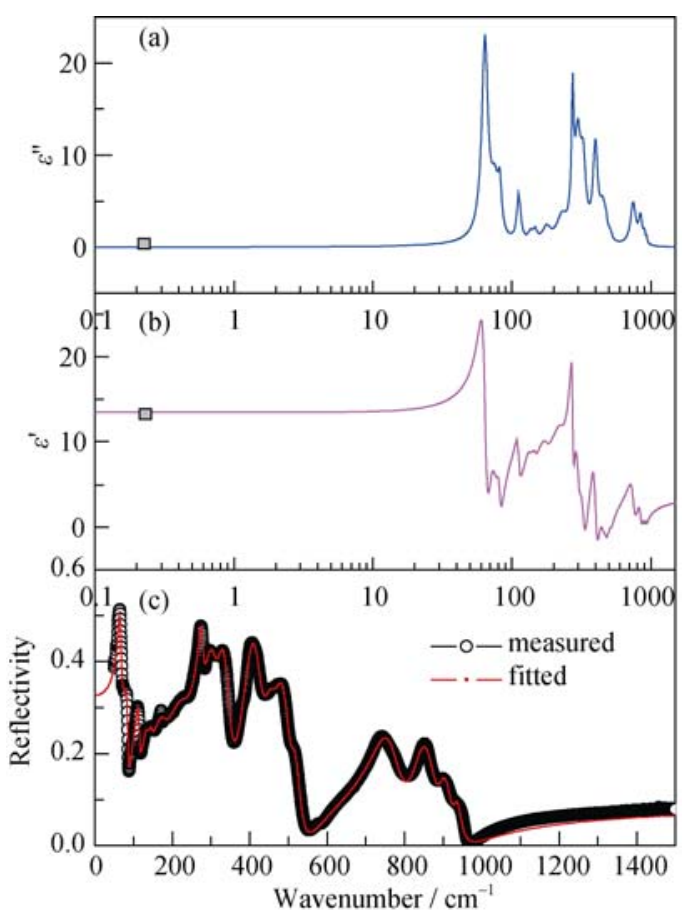

图 $5780^{\circ} \mathrm{C}$ 烧结所得 $\mathrm{BiMg}_{2} \mathrm{VO}_{6}$ 陶瓷的介电常数虚部(a)、介 电常数实部(b)、拟合和实测的红外反射谱(c)

Fig. 5 Imaginary part of complex permittivity(a), real part of complex permittivity (b) and fitted and measured infrared reflectivity spectra (c) of $\mathrm{BiMg}_{2} \mathrm{VO}_{6}$ ceramic sintered at $780^{\circ} \mathrm{C}$

$42000 \mathrm{GHz}$ ，大约为实际测量值的 2.7 倍。由于各种 缺陷造成的非本征损耗的影响, 实际陶瓷材料的品 质因数往往低于计算值 ${ }^{[12]}$ 。

\section{3 结论}

通过传统的固相反应法制备了单相 $\mathrm{BiMg}_{2} \mathrm{VO}_{6}$ 陶瓷。当烧结温度为 $720 \sim 840^{\circ} \mathrm{C}$ 时, $\mathrm{BiMg}_{2} \mathrm{VO}_{6}$ 陶瓷 的相对密度高达 $93.8 \% \sim 98.1 \%$ 。在 $780^{\circ} \mathrm{C}$ 条件下烧 结 $2 \mathrm{~h}$ 得到的 $\mathrm{BiMg}_{2} \mathrm{VO}_{6}$ 陶瓷微波介电性能最优: 介电常数 13.4, $Q \times f$ 值 $15610 \mathrm{GHz}(f=8.775 \mathrm{GHz})$, 温度系数为 $-87.2 \times 10^{-6} /{ }^{\circ} \mathrm{C}$ 。其光频介电常数和微波 介电常数分别为 3.4 和 13.5 。低的烧结温度和与 $\mathrm{Ag}$ 的化学相容性表明, $\mathrm{BiMg}_{2} \mathrm{VO}_{6}$ 有望用作新的低 温共烧陶瓷。

\section{参考文献:}

[1] SEBASTIAN M T, JANTUNEN H. Low loss dielectric materials for LTCC applications: a review. Int. Mater. Rev, 2008, 53(2): $57-90$.

[2] WANG NING, ZHAO MEI-YU, YIN ZHI-WEN. Low-temperature firing in microwave dielectric ceramics. J. Inorg. Mater, 2002, 17(5): 915-924. 
[3] LI YUE-MING, SONG TING-TING, YOU YUAN, et al. Research on low-temperature sintering of $\mathrm{Ca}_{0.3}\left(\mathrm{Li}_{1 / 2} \mathrm{Sm}_{1 / 2}\right)_{0.7} \mathrm{TiO}_{3}$ microwave dielectric ceramics. J. Inorg. Mater., 2008, 23(6): 1293-1297.

[4] GOLONKA L J. Technology and applications of low temperature cofired ceramic (LTCC) based sensors and microsystems. B Pol. Acad. Sci. Tech., 2006, 54(2):221-231.

[5] VALANT M, SUVOROV D. Chemical compatibility between silver electrodes and low-firing binary-oxide compounds: conceptual study. J. Am. Ceram. Soc., 2000, 83(11):2721-2729.

[6] ZHOU D, RANDALL C A, WANG H, et al. Microwave dielectric ceramics in $\mathrm{Li}_{2} \mathrm{O}-\mathrm{Bi}_{2} \mathrm{O}_{3}-\mathrm{MoO}_{3}$ system with ultra-low sintering temperatures. J. Am. Ceram. Soc., 2010, 93(4):1096-1100.

[7] ZHOU D, RANDALL C A, BAKER A, et al. Dielectric properties of an ultra-low-temperature cofiring $\mathrm{Bi}_{2} \mathrm{Mo}_{2} \mathrm{O}_{9}$ multilayer. $\mathrm{J}$. $\mathrm{Am}$. Ceram. Soc. 2010, 93(5):1443-1446.

[8] ZHOU D, QU W G, RANDALL C A, et al. Ferroelastic phase transition compositional dependence for solid-solution $\left[\left(\mathrm{Li}_{0.5} \mathrm{Bi}_{0.5}\right)_{x} \mathrm{Bi}_{1-x}\right]$ $\left[\mathrm{Mo}_{x} \mathrm{~V}_{1-x}\right] \mathrm{O}_{4}$ scheelite-structured microwave dielectric ceramics. Acta Mater, 2011, 59(4):1502-1509.

[9] ZHOU D, PANG L X, WANG H, et al. Phase transition, Raman spectra, infrared spectra, band gap and microwave dielectric properties of low temperature firing $\left(\mathrm{Na}_{0.5 x} \mathrm{Bi}_{1-0.5 x}\right)\left(\mathrm{Mo}_{x} \mathrm{~V}_{1-x}\right) \mathrm{O}_{4}$ solid solution ceramics with scheelite structure. J. Mater. Chem., 2011, 21:18412-18420.

[10] ZHOU D, WANG H, YAO X, et al. Phase transformation in
$\mathrm{BiNbO}_{4}$ ceramics. Appl. Phys. Lett., 2007, 90:172910.

[11] ZHOU D, PANG L X, YAO X, et al. Influence of sintering process on the microwave dielectric properties of $\mathrm{Bi}\left(\mathrm{V}_{0.008} \mathrm{Nb}_{0.992}\right) \mathrm{O}_{4}$ ceramics. Mater. Chem. Phys., 2009, 115:126-131.

[12] WANG QIU-PING, LIU HAN-CHEN. Study on far-infrared reflectivity spectra of microwave dielectric ceramic $(\mathrm{RbBi})_{1 / 2} \mathrm{MoO}_{4}$. Spectroscopy and Spectral Analysis, 2012, 32(9):2382-2384.

[13] XUN X, UMA S, YOKOCHI A, et al. Synthesis and structure of new $\mathrm{BiMn}_{2} \mathrm{MO}_{6}$ compounds where $\mathrm{M}=\mathrm{P}$, As, or V. J. Solid State Chem., 2002, 167: 245-248.

[14] HUANG J, SLEIGHT A W. Synthesis, crystal structure, and optical properties of a new bismuth magnesium vanadate: $\mathrm{BiMg}_{2} \mathrm{VO}_{6} . J$. Solid State Chem., 1992, 100:170-178.

[15] RADOSAVLJEVIC I, SLEIGHT A W. Variable temperature X-Ray diffraction study of bismuth magnesium vanadate, $\mathrm{BiMg}_{2} \mathrm{VO}_{6} . J$. Solid State Chem., 2000, 149:143-148.

[16] GUO R, ZHANG G K, LIU J. Preparation of Ag/AgCl/BiMg $\mathrm{VO}_{6}$ composite and its visible-light photocatalytic activity. Mater. Res. Bull., 2013, 48:1857-1863.

[17] LI GUANG-YAO. Low-temperature Sintering of $\mathrm{ZnNb}_{2} \mathrm{O}_{6}$ Ceramics. Xi'an: Master Thesis of Northwestern Polytechnical University, 2004.

[18] MAO MIN-MIN. Structures and Microwave Dielectric Characteristics of $\mathrm{SrRAlO}_{4}$-based ( $\mathrm{R}=\mathrm{La}, \mathrm{Nd}, \mathrm{Sm}$ ) Ceramics. Hangzhou: PhD Thesis of Zhejiang University, 2011. 